\title{
Gaseous liver abscess with Clostridium perfringens sepsis in a patient with neutropaenia
}

\author{
Waseem Amjad ${ }^{1}$, Su Chung ${ }^{1}$, Mirrah Mumtaz ${ }^{2}$, Ali Farooq ${ }^{3}$, Nasir Gondal ${ }^{1}$ \\ ${ }^{1}$ Department of Medicine, Northwell-Long Island Jewish Forest Hills Hospital, New York, USA \\ 2Department of Medicine, Albany Medical Centre, New York, USA \\ ${ }^{3}$ Charleston Area Medical Centre, Charleston, West Virginia, USA
}

Gastroenterology Rev 2019; 14 (2): 160-161

DOI: https://doi.org/10.5114/pg.2019.85902

\begin{abstract}
Address for correspondence: Waseem Amjad MD, Department of Medicine, Northwell-Long Island Jewish Forest Hills Hospital, New York, USA, phone: +1 9292457724, e-mail: waseemonline001@gmail.com
\end{abstract}

Clostridium perfringens is an anaerobic Gram-positive rod found in the gastrointestinal tract of humans and in soil. It can cause gas gangrene, sepsis, food poisoning, and necrotising enterocolitis. It gains access to the blood stream via a penetrating wound, or through mucosal defect in hepatobiliary system, gastrointestinal tract and female genital tract [1]. Clostridium perfringens is an unusual pathogen to cause liver abscess. We are presenting a fatal case of gaseous liver abscess with septic shock in the absence of penetrating trauma in the setting of neutropaenia.

A 77-year-old male with past medical history of diabetes mellitus, iron overload (idiopathic), atrial fibrillation (on rivaroxaban), myelodysplastic syndrome on chemotherapy (azacytidine subcutaneous injections) presented with fever for 3 days. Fever was associated with chills, shortness of breath, and confusion. The patient was febrile with a temperature of $104.9^{\circ} \mathrm{F}$, tachycardia $115 \mathrm{bpm}$, normotensive, and with tachypnoea 30 breaths/min. He was intubated for work of breathing and protection of airway. On examination, the patient was sedated and unarousable; the abdominal exam showed hepatomegaly and hypoactive bowel sounds.

On labs, haemoglobin $(\mathrm{Hb})$ was unreportable because of severe haemolysis; leukocytes count was 3,500/ $\mu \mathrm{l}$, absolute neutrophil count (ANC) was 105,000/ $\mu \mathrm{l}$, serum lactate was $6.4 \mathrm{mmol} / \mathrm{l}$, lactate dehydrogenase (LDH) was $6429 \mathrm{U} / \mathrm{l}$, and liver enzymes were elevated. Arterial blood gas post intubation showed $\mathrm{pH}$ 7.11, $\mathrm{pCO}_{2} 30 \mathrm{~mm} \mathrm{Hg}, \mathrm{pO}_{2}$ was $131 \mathrm{~mm} \mathrm{Hg}$ on $40 \%$ $\mathrm{O}_{2}$, and $\mathrm{HCO}_{3} 13 \mathrm{mmol} / \mathrm{l}$. Computed tomography (CT) showed $2.8 \times 1.9 \mathrm{~cm}^{2}$ complex air collection in the right hepatic lobe (Figure 1$)$.

The patient was given aggressive hydration. He received 2 units of packed red blood cells (PRBC) based on suspicion of anaemia due to severe haemolysis. His blood pressure dropped to $78 / 36 \mathrm{~mm} \mathrm{Hg}$ in the next few hours. He was started on broad-spectrum antibiotics: cefepime, vancomycin, and metronidazole, based on suspicion of liver abscess and sepsis. The patient was started on a norepinephrine and sodium bicarbonate drip. Repeat labs showed worsening of liver function tests (LFTs), renal functions, lactate, lactate dehydrogenase (LDH), and metabolic acidosis. Multiple blood specimens were noted to be haemolysed. Lab estimated haemoglobin $(\mathrm{Hb})$ level was $8.3 \mathrm{~g} / \mathrm{dl}$ after 2 units of PRBC. Haemoglobin dropped further during the hospital stay. He received 3 more units of PRBC, 3 units of fresh frozen plasma (FFP), and one unit of platelets.

Blood culture grew Gram-positive rods of Clostridium perfringens. The patient's condition worsened during the hospital stay and he developed multiorgan failure. He was kept on ventilator and pressor support. He developed cardiac arrest on the second day of admission. Return of spontaneous circulation was achieved after 4 min of resuscitation. The family signed a do not resuscitate (DNR) form, and the patient expired the same day.

Clostridium perfringens sepsis is one of the most fulminant bacterial infection in humans. There are five types of $C$. perfringens from $A$ to $E$, based on the type of exotoxin produced. Alpha-type toxin (phospholipase C) is the most common toxin involved in gas gangrene. Alpha-toxin hydrolyse phospholipids, phosphorylcholine, and diglyceride lead to haemolysis and tissue damage $[1,2]$. The Simon et al. review of $C$. perfringens cases with haemolysis shows a high mortality rate of $74 \%$ with median time to death of $9.7 \mathrm{~h}$ [3].

Risk factors for $C$. perfringens septicaemia include old age, poorly controlled diabetes, cirrhosis, chemotherapy-induced neutropaenia, and malignancies [4]. In myelodysplastic syndrome (MDS) patients, neutropae- 


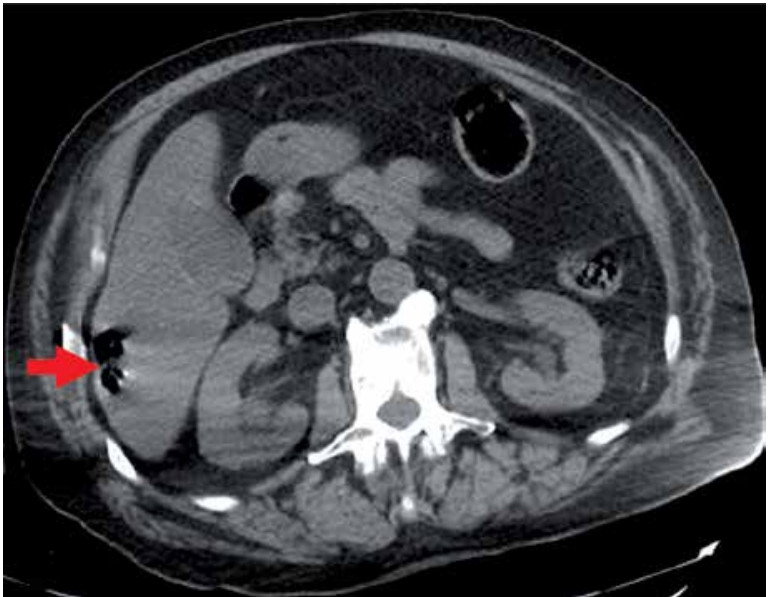

Figure 1. Computed tomography abdomen axial view shows complex air collection in right hepatic lobe (red arrow)

nia is one of the major risk factors for developing infections. A study by Zahar et al. showed that haematological malignancies and intra-abdominal tumours can be associated with anaerobic bacteraemia. This study also revealed that $33 \%$ of individuals who developed anaerobic bacteraemia had ANC $<500 \times 10^{3} / \mu \mathrm{l}[5]$.

Liver abscess is the most common type of the visceral abscess and is associated with significant mortality of $20 \%$. The most common organisms causing liver abscess are Entamoeba, Klebsiella, Escherichia coli, and Streptococcus [6]. Gas-forming liver abscess accounts for $7 \%$ to $24 \%$ of pyogenic liver abscesses. It has high mortality despite management [7]. Most common aetiology of liver abscess is biliary disease; other common sources are contiguous spread from colon, kidney, lung, or stomach, penetrating trauma, and a large number are cryptogenic. Only a few cases of $C$. perfringens liver abscess with sepsis are found in the literature, few are post-operative, and one developed secondary to gastroenteritis [8]. Our case is unique because the patient developed gaseous abscess with $C$. perfringens bacteraemia in the presence of neutropaenia. There was no perforation trauma or surgery. There are remote chances that the bacteria entered the blood stream during the subcutaneous chemotherapy injections.

Presenting complaints are nonspecific, including fever, abdominal pain, jaundice, confusion, and crepitus (if gas gangrene is present). In most cases liver abscess is diagnosed by a CT scan of the abdomen [4]. Intravenous penicillin is the treatment of choice. Other management includes blood transfusion as needed and correction of metabolic derangements. Immediate use of antibiotics is associated with better survival $[1,9]$. Clostridium perfringens is susceptible to carbapenems, penicillin, metronidazole, clindamycin, rifampicin, and tetracycline. Stevens et al. showed that clindamycin and metronidazole have rapid activity against $C$. perfringens [10]. Our patient was haemodynamically unstable and was not a candidate for surgery. He did not respond well to broad-spectrum antibiotics and resuscitation possibly because of an underlying immunocompromised state. Surgical drainage of the abscess can improve the survival, but extensive infection in a haemodynamically unstable patient and multiple comorbidities can limit the surgical intervention [9].

In patients with neutropaenic fever, empiric treatment for anaerobic bacteria should be considered earlier. Clostridium perfringens can occasionally cause liver abscess with sepsis, which has high mortality despite management. In patients with liver abscess, early surgical drainage can improve the survival.

\section{Conflict of interest}

The authors declare no conflict of interest.

\section{References}

1. Hashiba M, Tomino A, Takenaka N, et al. Clostridium perfringens infection in a febrile patient with severe hemolytic anemia. Am J Case Rep 2016; 17: 219-23.

2. Kuroda S, Okada Y, Mita M, et al. Fulminant massive gas gangrene caused by Clostridium perfringens. Intern Med 2005; 44: 499-502.

3. Simon TG, Bradley J, Jones A, Carino G. Massive intravascular hemolysis from Clostridium perfringens septicemia: a review. J Intensive Care Med 2014; 29: 327-33.

4. Law ST, Lee MK. A middle-aged lady with a pyogenic liver abscess caused by Clostridium perfringens. World I Hepatol 2012; 4: 252-5.

5. Zahar JR, Farhat H, Chachaty E, et al. Incidence and clinical significance of anaerobic bacteraemia in cancer patients: a 6-year retrospective study. Clin Microbiol Infect 2005; 11: 724-9.

6. Jha AK, Das A, Chowdhury F, et al. Clinicopathological study and management of liver abscess in a tertiary care center. J Nat Sci Biol Med 2015; 6: 71-5.

7. Chou FF, Sheen-Chen SM, Chen YS, Lee TY. The comparison of clinical course and results of treatment between gas-forming and non-gas-forming pyogenic liver abscess. Arch Surg 1995; 130: 401-5.

8. Eltawansy SA, Merchant C, Atluri P, Dwivedi S. Multi-organ failure secondary to a Clostridium perfringens gaseous liver abscess following a self-limited episode of acute gastroenteritis. Am J Case Rep 2015; 16: 182-6.

9. van Bunderen CC, Bomers MK, Wesdorp E, et al. Clostridium perfringens septicaemia with massive intravascular haemolysis: a case report and review of the literature. Neth J Med 2010; 68: 343-6.

10. Stevens DL, Maier KA, Mitten JE. Effect of antibiotics on toxin production and viability of Clostridium perfringens. Antimicrob Agents Chemother 1987; 31: 213-8.

Received: 4.07.2017

Accepted: 19.02.2019 